\title{
Target-like chondrocytes with thick perichondrocytic rings in cartilage-forming tumours. Preliminary report
}

\author{
Ctibor Povysil ${ }^{a}$, Martin Kana ${ }^{b}$
}

\begin{abstract}
Background. Here we present our experience with the occurrence of neoplastic chondrocytes with target-like appearance surrounded with unusual hypertrophic thick eosinophilic perichondrocytic rings (baskets), sometimes containing two or several layers.

Methods and Results. Pericellular rings (baskets) were positive in APAS and Masson's staining method and showed immunoreactivity with antibody against type IV collagen. Such single cells or small groups of such cells were observed rarely in 3 osteochondromas, 2 skeletal chondromas, 2 extraskeletal chondromas and 2 skeletal and 1 laryngeal chondrosarcomas. Moreover, 1 unusual soft tissue chondrosarcoma was composed entirely of target-like chondrocytes with hypertrophic extremely thick perichondrocytic rings. Such cartilage-forming tumour with target-like cells, which, to the best of our knowledge, is the first such chondrosarcoma reported in the literature.

Ultrastructural evidence is presented that perichondrocytic rings have complicated structure. They contained microfibrillar component with abundant admixture of irregular aggregates of dense amorphous non-fibrillar material localised in lacunar spaces. In outer part of the rings predominated microfibrillar structures corresponding to type VI collagen that produced a rather dense capsule-like demarcation line against surrounding intercellular spaces.

Conclusions. The described unusual changes are probably the result of hyperproduction and remodelation of perichondrocytic matrix by abnormal neoplastic chondrocytes in response to unknown factors. Local vascular and molecular signals, may be supposed as probable causes of this phenomenon.
\end{abstract}

Key words: cartilage tumours, target-like chondrocyte, chondrocytes with thick APAS positive pericellular rings, immunohistochemistry, electron microscopy

Received: March 9, 2021; Revised: April 6, 2021; Accepted: April 14, 2021; Available online: April 27, 2021

https://doi.org/10.5507/bp.2021.024

(c) 2021 The Authors; https://creativecommons.org/licenses/by/4.0/

alnstitute of Pathology, 1st Faculty of Medicine, Charles University and General Medical Hospital, and Institute of Postgraduate Studies, Prague, Czech Republic

${ }^{b}$ Department of Otorhinolaryngology and Head and Neck Surgery, 1st Faculty of Medicine, Charles University and University Hospital Motol, Prague, Czech Republic

Corresponding author: Ctibor Povysil, e-mail: ctibor.povysil@lf1.cuni.cz

\section{INTRODUCTION}

Articular non-neoplastic chondrocytes as well as tumour chondrocytes are situated in lacunar spaces surrounded with a thin ring composed of type VI collagen fibres $^{1,2}$. The collagen in the pericellular matrix shows the basket-like morphology ${ }^{3}$. This unique region is called "pericellular matrix" (PCM) that integrates with surrounding tissue via the "periterritorial matrix" connecting the PCM to the "interterritorial matrix". Together the chondrocyte and its PCM have been termed the "chondron"3. Chondrons are primary functional and metabolic unit of cartilage protecting the integrity of the chondrocytes $^{4,5}$. The chondrons represent a distinct functional compartment within articular cartilage. Cartilage matrix turnover is a slow, continuous process. As the PCM surrounds every chondrocyte, all matrix components secreted by chondrocytes must pass through this region ${ }^{3,5}$. We suppose that similar situations are also possible to occur in conventional cartilage-forming tumours. We know, on the basis of our experience, that rings of majority neoplastic chondrocytes are thin and usually contain only sparse collagen fibrils ${ }^{6-8}$.

During the review of our files containing 500 chondrogenic tumours we recognised, that neoplastic cell population was not homogeneous and in some tumours contained small component of cells with unusual thick APAS positive perichondrocytic rings. In this preliminary report we present our experience with this rare phenomenon, that will be described in details in our next study describing chondrogenic tumours completely composed of such unusual cells. Here we shall present only basic morphological characteristics of neoplastic so-called target-like chondrocytes. Because of the rarity of such unusual histological pattern of chondrogenic tumours, that was probably not described in the literature, we present our first histological, immunohistochemical and electron microscopic findings. 


\section{MATERIAL AND METHODS}

A review of the 500 chondrogenic tumours from the bone and soft tissue files of Institute of Pathology of 1st Medical Faculty and General Medical Hospital in Prague and a review of consulting files of the author of this article were undertaken. Haematoxylin and eosin stained slides were available in all cases. We identified and selected 3 osteochondromas, 2 skeletal and 2 extraskeletal chondromas and 3 chondrosarcomas that contained an admixture of target-like neoplastic chondrocytes with thick perichondrocytic rings. Moreover, 1 soft tissue chondrosarcoma entirely composed of target-like chondrocytes was recognised and examined.

In some cases we completed staining with Alcian blue and Safranin O that enabled evaluating the content of glycosaminoglycans. Masson trichrome, Gomori impregnation technique and Sirius red was used for demonstration of collagen fibres. Picrosirius red detected collagen-type I to V collagen. PAS staining in diastase treated sections showed tissue carbohydrates. Immunohistochemical examination with antibodies against S-100 protein, D2-40, type VI and II collagen was performed in three cases.

Electron microscopic findings in cartilaginous tumours were reviewed from our files of bone tumours, that were summarised in our monographs $s^{6,7}$. In soft tissue chondrosarcoma entirely composed of target- like tumour chondrocyte we used formalin fixed samples from paraffin-embedded tissue, because glutaraldehyde-fixed material was not available. Ultrathin section were examined under JEM -1400 Plus Jeol electron microscope. Furthermore, samples were analysed by transmission electron microscopy and these findings were compared with our ultrastructural findings in the group of different types of chondrogenic tumours examined and reported previously in our laboratory ${ }^{6-8}$.

\section{RESULTS}

\section{Microscopical examination}

Histologically we identified 10 cases in total with focal occurrence of target-like chondrocytes ( 3 osteochondromas, 2 skeletal and 2 extraskeletal chondromas and 2 skeletal and 1 laryngeal chondrosarcomas). These unusual cells occurred as single elements (Fig. 1 and 2), sometimes in areas with calcification of matrix (Fig. 2), or they formed small groups in tumour hyaline cartilage matrix (Fig. 3 and 4). In one case of skeletal chondrosarcoma, these cells dominated in one tumour lobules (Fig. 3), while in other lobules of this tumour occurred usual cell population with thin perichondrocytic rings. However, peripheral part of some lobules again contained irregular layer of chondrocytes with thick rings (Fig. 4). Moreover, 1 soft tissue chondrosarcoma was completely composed of target-like cells with extremely thick pericellular rings (Fig. 5 and 6), however, it will be described in details in our next presentation.

Perichondrocytic rings had homogenous structure or were composed of two or several concentric hyalin lay-

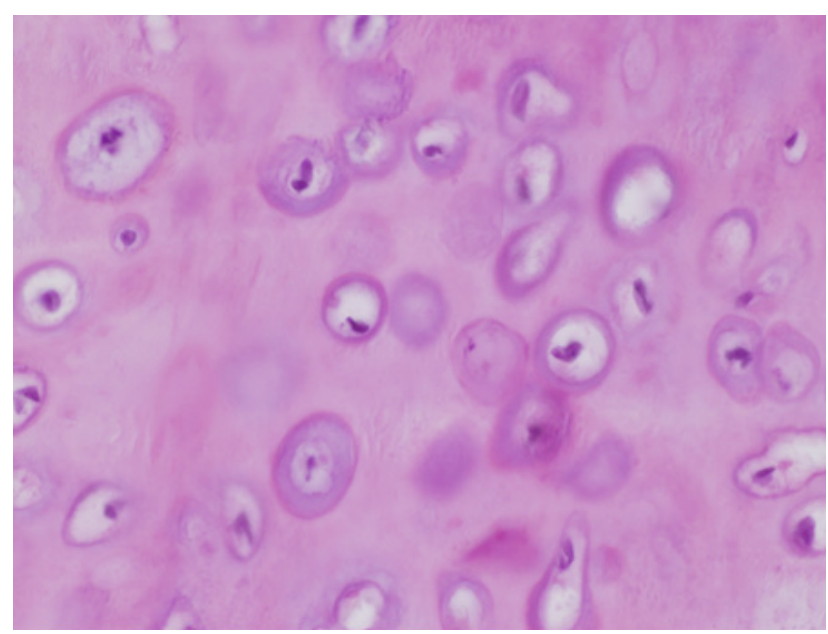

Fig. 1. Detail of several malignant chondrocytes with thick perichondrocytic rings as they were observed in mesenchymal chondrosarcoma. HE, x100.

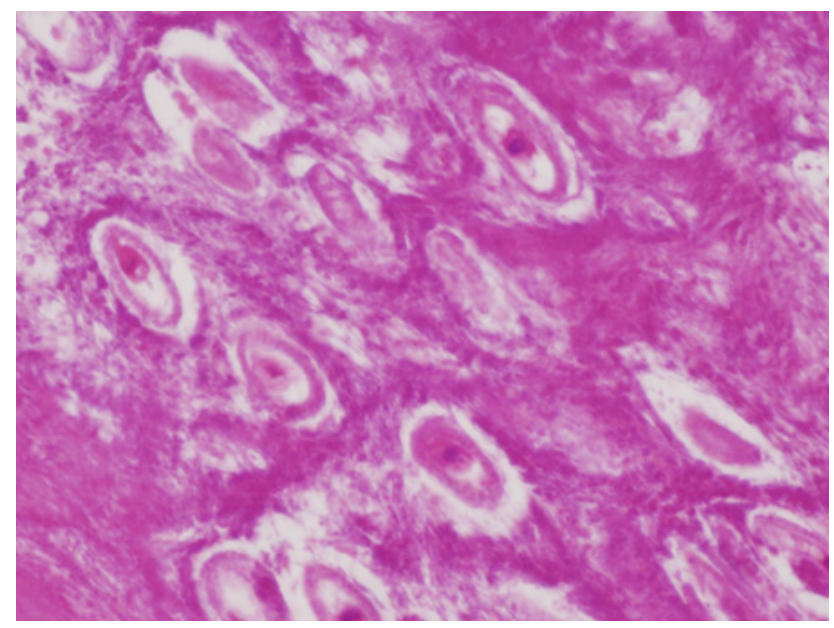

Fig. 2. Target-like chondrocytes in calcified periosteal chondroma. HE, x200.

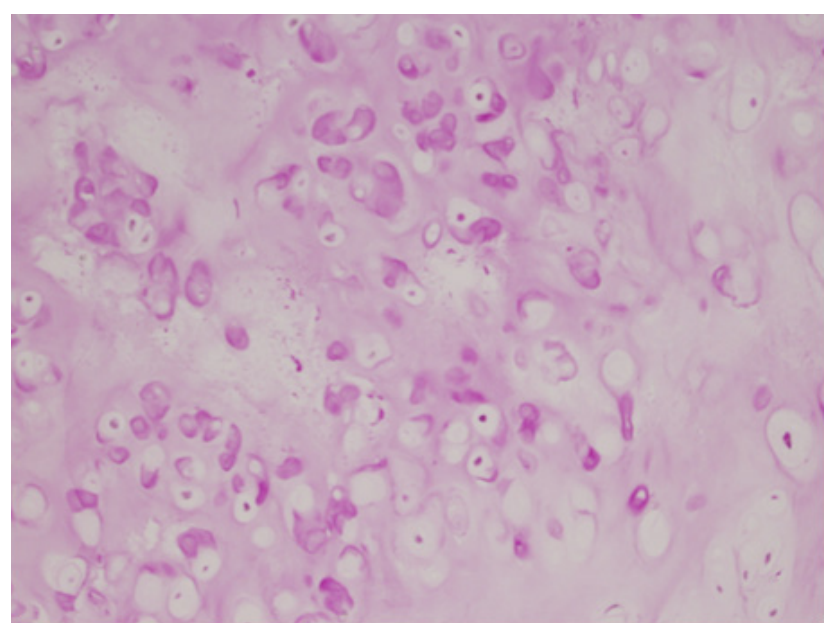

Fig. 3. Skeletal conventional chondrosarcoma contained one neoplastic lobule predominantly composed of malignant chondrocytes with APAS positive thick rings, $\mathrm{x} 50$. 


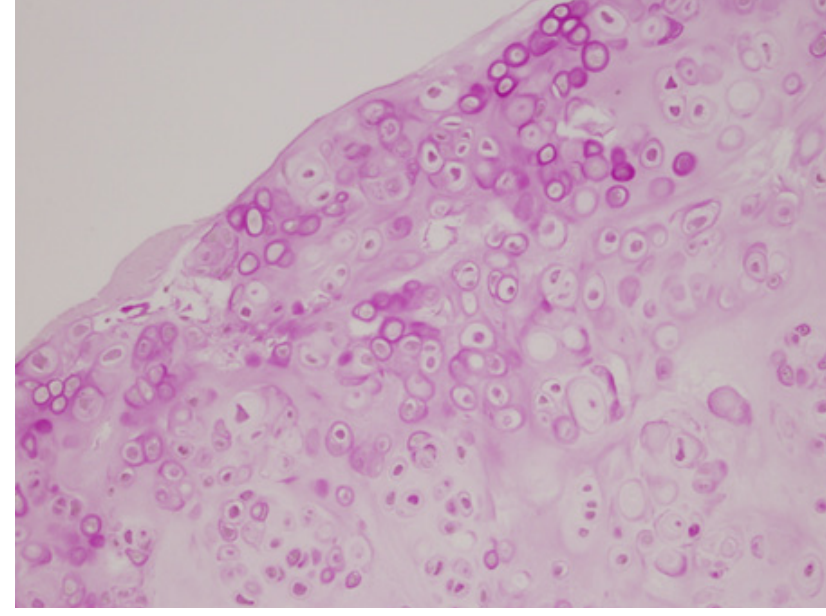

Fig. 4. Skeletal conventional chondrosarcoma also contained chondrocytes with thick APAS positive rings at the periphery of some neoplastic lobules, $\mathrm{x} 50$.

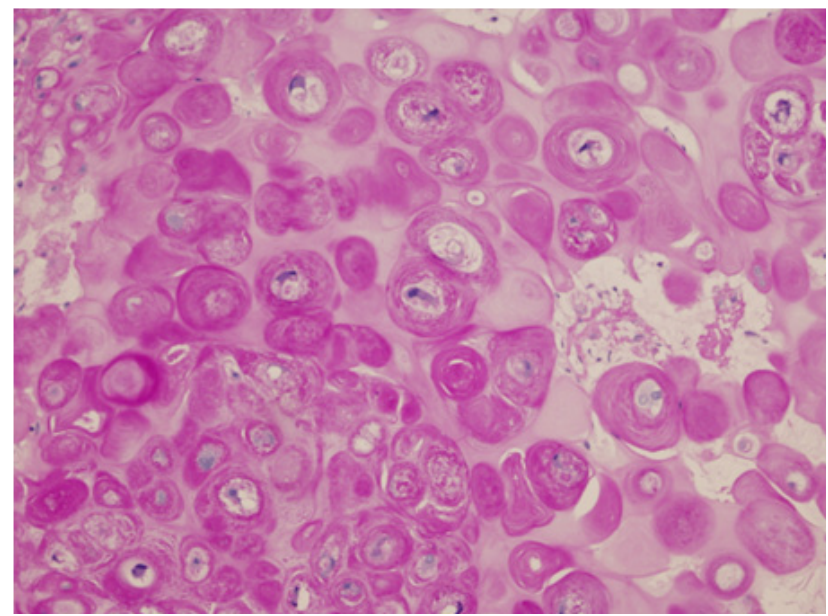

Fig. 6. Detail of target- like chondrocytes with thick APAS positive rings that form the majority of cell population in soft tissue chondrosarcoma from previous picture. APAS, x100.

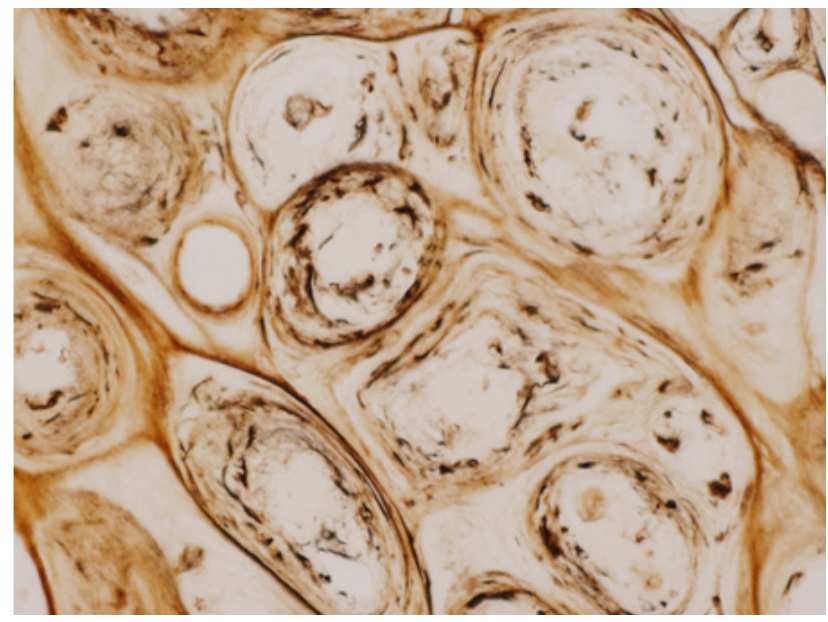

Fig. 8. Gomori's method impregnated sparse collagen fibres, $\mathrm{x} 200$.

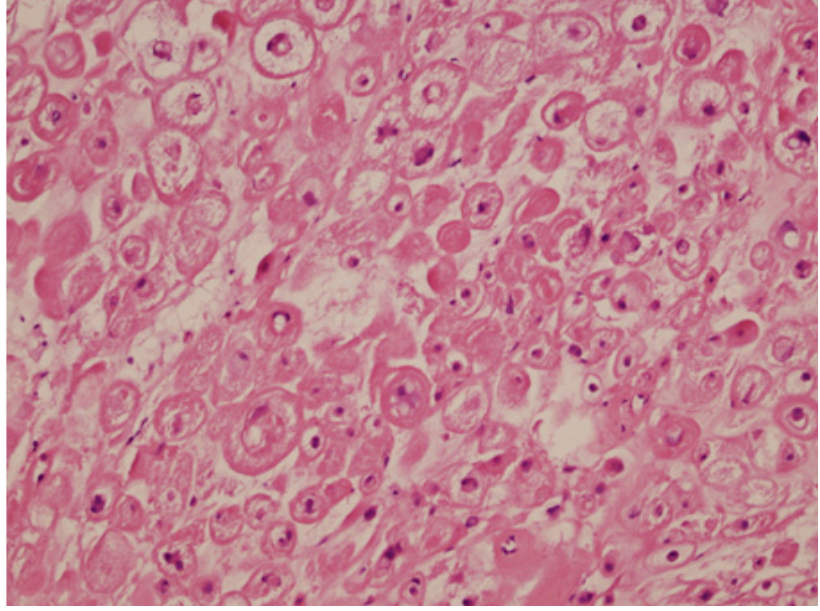

Fig. 5. Chondrosarcoma of soft tissue, that contained only target-like malignant chondrocytes in all its parts. HE, x50.

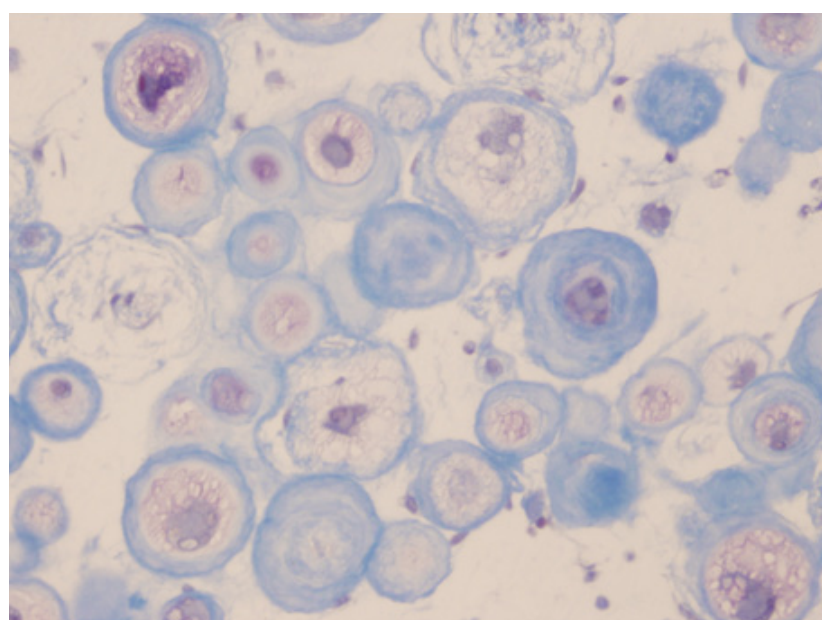

Fig. 7. Detail of target-like chondrocytes in Masson's staining method demonstrating the presence of collagen, $\mathrm{x} 200$.

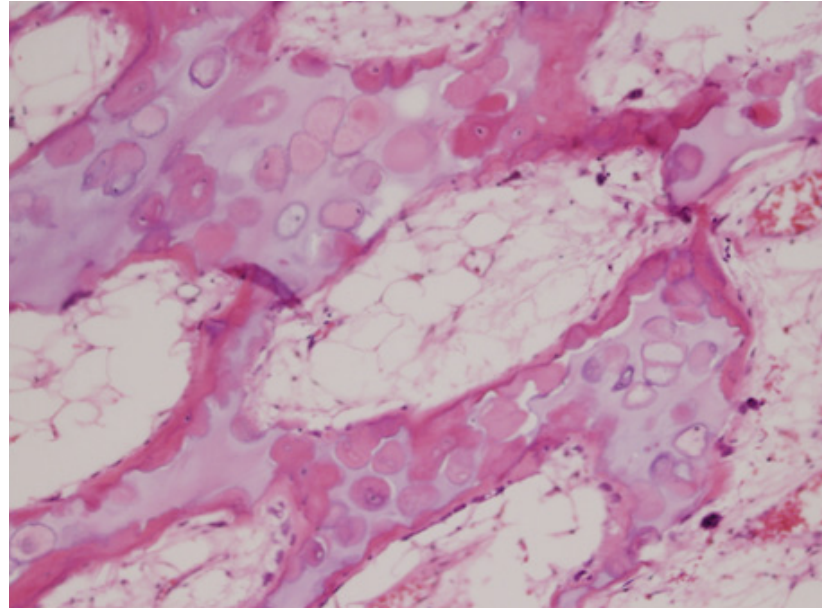

Fig. 9. Target-like chondrocytes in bone trabeculae of the stalk of osteochondroma.HE, x100. 
ers showing APAS positivity (Fig.3, 4 and 6). The most intensive APAS positivity usually occurs in outer compact zone of ring in contrast to inner layer that stained weak. Moreover, in some lacunar spaces between the chondrocytes bodies and the rings occurred small aggregates of APAS positive material. These fragmented aggregates of APAS positive material formed the majority of the perichondrocytic mass in some chondrons. In Masson's trichrom method the rings had more compact structure and stained as collagen (Fig. 7). In Gomori method there occurred single impregnated fibres in these rings (Fig. 8). Some lacunar spaces lacked cells and were completely substituted by APAS and collagen positive acellular tissue forming together with rings compact round formation, corresponding to globally sclerotic chondrons (Fig. 6). Other specific methods could not be used in our study, because the occurrence of such cells was only sporadic.

We also observed similar cells in bone trabeculae of stalk of osteochondromas. The persisting cartilage in incompletely ossified central part of bone trabeculae contained some chondrons with thick perichondrocytic rings, but in other elements was difficult to differentiate these cells from osteocytes with osteoid rings (Fig. 8 and 9).

Basic immunohistochemical examinations showed that a majority of the tumour cells reacted strongly diffusely or focally with the marker against vimentin, osteonectin, podoplanin and S-100 protein. The staining with antibody against Ki67 showed minimal proliferative activity of neoplastic chondrocytes in chondromas as well as in chondrosarcomas.

Immunoreactivity of type VI collagen in the pericellular rings (Fig. 10) and type II collagen in the extracellular interterritorial matrix was recognized. Antibody against type VI collagen stained intensively peripheral parts of the rings, small aggregates in interterritorial regions and in globally sclerotic acellular chondrons positivity was also observed.

Electron micrographs revealed many relatively normal appearing chondrocytes with intracytoplasmic organelles such as rough endoplasmic reticulum, mitochondria, glycogen granules and lipid droplets. The chondrocytes of different shape were deposited in round lacunar spaces containing small amount of proteoglycan granules and irregular aggregates of the amorphous dense material of different size and shape (Fig. 11). The dense material resembled dense product accumulated sometimes in dilated rough endoplasmic reticulum observed regularly in the cytoplasm of neoplastic chondrocytes examined in our laboratory. The outer zone of pericellular ring was formed by the layer of condensed thin collagen fibrils with an admixture of less dense trabecullar material oriented parallel to the chondrocytes and producing capsulelike demarcation line against surrounding intercellular spaces (Fig. 11). Matrix of intercellular spaces contained identical thin collagen fibrils with irregular orientation in the form of network. In the spaces between microfibrils there were small dense granules corresponding to

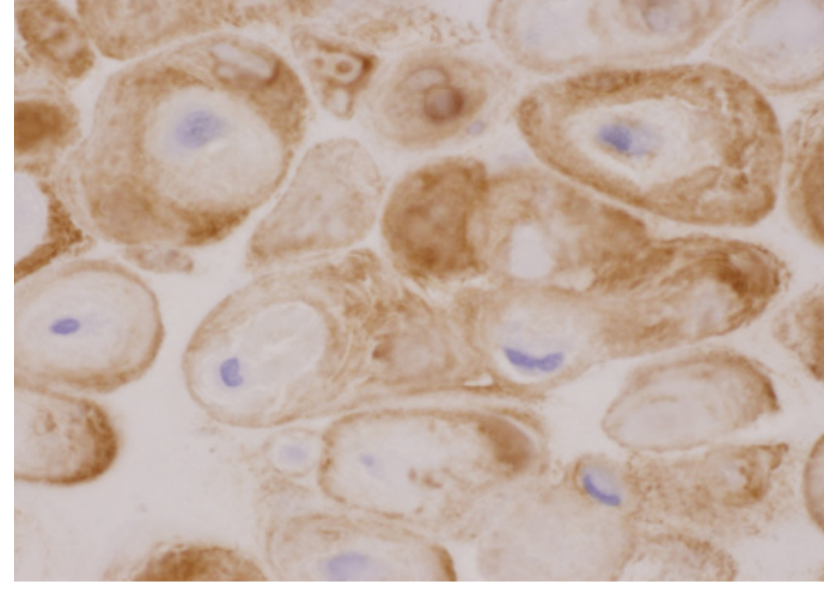

Fig. 10. Positivity of type VI collagen in pericellular rings of tumour chondrocytes, $\mathrm{x} 200$.

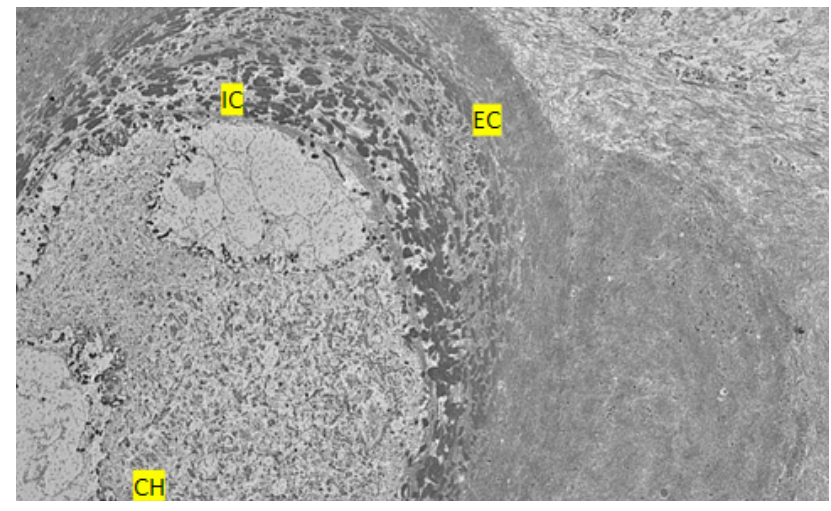

Fig. 11. Electron microscopy of neoplastic target-like chondrocyte from unusual soft tissue chondrosarcoma. Chondrocyte $(\mathrm{CH})$ is surrounded with thick ring composed of internal (IC) and external EC) component together producing thick capsule. Both components differed in their internal structures. Original magnification $\mathrm{x} 8000$.

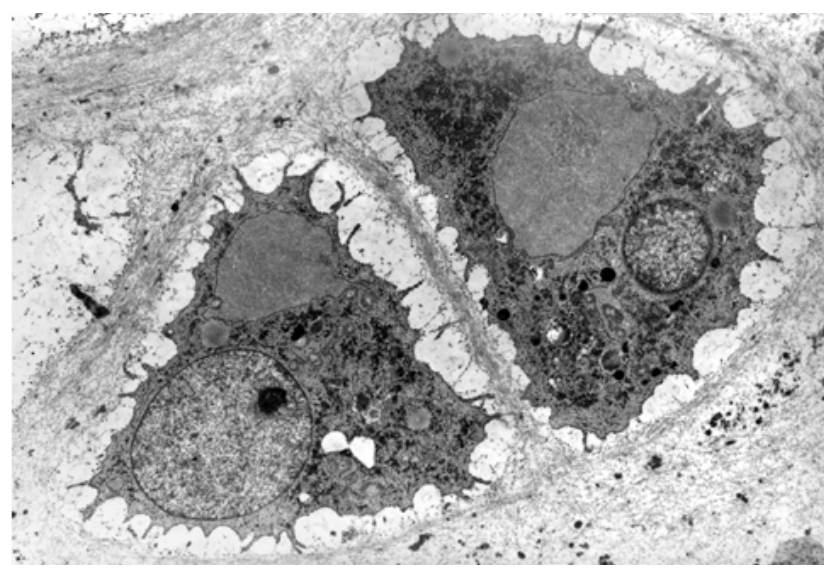

Fig. 12. Electron microscopy picture from conventional chondrosarcoma shoving usual neoplastic chondrocytes surrounded with normal thin capsule, containing only fibrillar component. Original magnification x3500. 
glycosaminoglycans (Fig. 11). Control figure from our chondrosarcoma files shows usual pattern of normal thin perichondrocytic ring occurring in conventional cartilage forming tumours (Fig. 12).

\section{DISCUSSION}

Cartilage-forming tumours are heterogeneous group of tumours with well known histological features ${ }^{6,8-11}$. The hallmark of all differentiated cartilaginous tumours is the presence of neoplastic chondrocytic cells producing characteristic cartilaginous tumour matrix. The tumour cells, embedded in the hyaline cartilage matrix, resemble normal chondrocytes of hyaline cartilage and lie in lacunar spaces sometimes surrounded with very thin rings. Cartilaginous tumours are the most common neoplasms affecting bone ${ }^{6,9-11}$. Conventional soft-tissue cartilage-forming tumours are predominantly benign tumours, with local recurrence rate of 17 to $18 \%$ (ref. ${ }^{11,12-16}$ ). They usually arise from soft tissue adjacent to tendons in the hands and feet of adults. They may exhibit variable degrees of cytological atypia, including enlarged cells ${ }^{15,16}$. Some soft-tissue chondromas show a striking resemblance to chondroblastoma of bone ${ }^{17}$ or contained lipoblast-like cells ${ }^{18}$. It is well known that these tumours may undergo secondary changes or exhibit features such as dystrophic calcification, endochondral ossification or focal myxoid degeneration or CPPD deposition ${ }^{19}$. Such changes may make the diagnosis more difficult. Histological structure of skeletal enchondromas is similar. Osteochondromas have well known classic histological picture with cartilaginous cap, and bony stalk, with endochondral ossification in the interface between cartilage and bone. Our chondrosarcomas had lobular pattern with heterogeneous composition of cell population containing rare target-like chondrocytes and the chondrocytes without pericellular rings predominated. From these finding is evident that tumour chondrocytes are phenotypically unstable. Basic immunohistochemical examination showed S-100 protein and podoplanin positivity of neoplastic chondrocytes as it is typical for cartilage cells $\mathbf{s}^{6,20,21}$. Positivity with antibody against collagen type VI confirmed that target-like tumour cells are surrounded with identical collagen fibres described in the rings of normal articular chondrocytes ${ }^{1}$, as well as in neoplastic chondrocytes, where regulates pericellular matrix properties ${ }^{2}$. However, their thickness was different.

During review of our collection of chondrogenic tumours we observed solitary neoplastic chondrocytes with thick perichondrocytic rings, that occurred sometimes in areas with calcification of the intercellular matrix. In one chondrosarcoma these cells formed the majority of neoplastic chondrocytes in one lobule of this neoplasm, that showed distinct APAS positivity. In one other cartilage forming soft tissue tumour the whole population of neoplastic cells was composed of chondrocytes with targetlike appearance. This unusual chondrogenic tumour will be described in our next publication in details, because it has some specific histological features and different genetic profile that probably represent specific subvariant of chondrosarcoma. However, we could specify the structural characteristic of the APAS positive rings that contained collagen type VI and on the ultrastructural level they were composed of microfibrils, proteoglycan granules and and irregular deposits of dense non-fibrillar material. We are proposing for such tumours the term „cartilage forming tumour with target-like chondrocytes containing hypertrophic pericellular rings.

Chondrocytes represent a unique cell type that can undergo dynamic multimorphological signals. It is well known that each step of chondrocytic differentiation pathway in developing endochondral bone is characterized by specific histological features, and gene expression profiles $^{22,23}$. They are regulated by complex and multifactorial signals ${ }^{23}$. That was a reason why we looked for the existence of similar cells with thick perichondrocytic rings in developing skeleton. Unfortunately, we found no information in the literature about the existence of cartilage cells with hypertrophic rings in normal chondrogenesis ${ }^{23,25}$. Review of growth plate cartilage in skeletal samples from our autoptic normal foetal bone tissue files finished also with negative results. However, information that hypertrophic chondrocytes occurring at the chondroosseous front in growth plate cartilage express EGFR1 and beta-catenin ${ }^{26}$ are interesting, but thickness of their pericellular rings was not changed. Similar situation with hypertrophic chondrocytes surrounded with normal perichondrocytic rings was also described in osteoarthritis ${ }^{27}$. Moreover, mutations in EGFRs cause several skeletal phenotypes such as limb defects, achondroplasia and craniosynostosis or they are associated with the development of some cartilage tumours ${ }^{28}$.

In summary, this is the first histological analysis describing the occurrence of neoplastic chondrocytes with unusual hypertrophic thick pericellular rings observed in soft tissue and bone cartilage-forming tumours. Analysis of the literature data and our files showed that cartilaginous tumours with the target-like chondrocytes is uncommon feature occurring rarely in limited number of cases. Histogenesis and etiopathogenesis of this phenomenon is poorly understood and the clinical significance is unclear. However, their existence confirmed the heterogeneity of neoplastic chondrocytes in some tumours. Our findings bring the first level of information about such unusual transformation of neoplastic chondrocytes that can predominate in some cartilage tumours and than histological pattern of chondromas or chondrosarcomas is completely modified, as we observed in one our neoplasm. We propose for such tumours the name "cartilage forming- tumour with target-like chondrocytes". Details of such tumours will be reported in our next work. Electron microscopy confirmed that perichondrocytic rings have complex structure composed of several types of various material produced by these phenotypically unusual neoplastic chondrocytes.

Acknowledgement: This work was supported by Ministry of Health, Czech Republic (Conceptual development of research organization 64165, General University Hospital 
in Prague), and by Charles University (Project Progress Q28/LF1).

Author contributions: CP: analysis of histological slides and immunohistochemical findings, manuscript writing, corresponding author; $\mathrm{MK}$ : electron microscopic examination, literature search, analysis of clinical data.

Conflicts of interest statement: The authors state that there are no conflicts of interest regarding the publication of this article.

\section{REFERENCES}

1. Söder S, Hambach L, Kissner R, Kirchner T, Aigner T. Ultrastructura localization of type $\mathrm{VI}$ collagen in normal adult and osteoarthritic human articular cartilage. Osteoarthritis Cartilage 2002;10(5):46470.

2. Zelinski NA, Leddy HA, Sanchez-Adams J, Zhang J, Bonaldo W, Liedtke W, Guilak F. Collagen VI regulates pericellular matrix properties, chondrocytes swelling and mechanotransduction in articular cartilage. Arthritis Rheumatol 2015;67(5):1286-94.

3. Wilusz RE, Sanchez-Adams J, Guilak F. The structure and function of the pericellular matrix of articular cartilage. Matrix Biol 2014;39(3):25-3219.

4. Poole CA, Flin MH, Beaumont BW. Chondrons in cartilage. Ultrastructural analysis of the pericellular microenviroment in adult human articular cartilage. J Orthoped Res 1987;5(4):509-22.

5. Poole CA. Review. Articular cartilage chondrons: form, fuction and failure. J Anat 1997;19(Pt1):11-13.

6. Povýšil C, Mařík I, Maříková A, Horák M. Pathomorphology of the bone and joint diseases (In Czech) Prague: Galén; 2017.

7. Povýšil C. Histopathology and ultrastructure of tumours and tumourlike lesions of bone: Acta Univ Carol Med Monogr 1986;116:1-204

8. Povýšil C, Matějovský Z. A comparative ultrastructural study of chondrosarcoma, chordoid sarcoma, chordoma and chordoma periphericum. Path Res Pract 1985;179(4-5):546-93.

9. Dorfman HD, Czerniak B. Bone tumors, 1st ed., St. Louis, Missouri: Mosby; 1998.

10. Bovée AM, Flanagan AJ, Lazar GP, Nielsen A, Yoshida J. WHO classification of tumours. Soft tissue and bone tumours. 3rd chapter: Bone tumours., 5th ed., Lyon: IARC; 2020.

11. Qasem SS, DeYoung BR. Cartilage-forming tumours. Semin Diagn Pathol 2014;31(1):10-20.

12. Dahlin DC, Salvador AH. Cartilaginous tumours of the soft tissues of the hands and feet. Mayo Clin Proc 1974;49(10):721-6.
13. Chung EB, Enzinger FM. Chondroma of soft part. Cancer 1978;41(4):1414-249.

14. Cardia R, Favazzi CM, Fenga D, Rosa MA, Leni A. A large extraskeletal chondroma: An unusual location in the lower extremity, huge extraskeletal chondroma: An unusual localization in the leg. J Orthop Case Rep 2019;9(1):74-7.

15. Makni SW, Makni SK, Triki FE, Mellouli M, Abid R, Kallel R, Charfi S, Boudawara TS. Well-differentiated extraskeletal chondrosarcoma: about a new case. Pathologica 2018;110(2):103-5.

16. Saito $M$, Nishimoto $K$, Nakayama R, Kikuta $K$, Nakamura $M$ Matsumoto $\mathrm{M}$, Morioka $\mathrm{H}$. Extraskeletal chondroma of the index finger. A case report. Case Rep Oncol 2017;10(2):479-84.

17. Cates JM, Rosenberg AE, O'Connell JX, Nielsen GP. Chondroblastomalike chondroma of soft tissue: an underrecognized variant and its differential diagnosis, Am J Surg Pathol 2001;25(5):661-66.

18. Chan JK, Lee KC, Saw D. Extraskeletal chondroma with lipoblast-like cells. Hum Pathol 1986;17(12):1285-7.

19. Athanasou NA, Caughey M, Burge P, Woods CG. Deposition of calcium pyrophosphate dihydrate crystals in soft tissue chondromas. Ann Rheum Dis 1991;50(12):950-2.

20. Daugaard S, Christensen LH, Hogdall H. Markers aiding the diagnosis of chondroid tumours: an immunohistochemical study including osteonectin, bcl-2, cox-2, actin, calponin D2-40 (podoplanin), mdm2, CD117(c-kit), and YKL-40, APMIS 2009;117(7):518-25.

21. Ordonez NG. Podoplanin: a novel diagnostic immunohistochemical marker. Adv Anat Pathol 2006;13(2):83-88.

22. Lefebvre V, Smits P. Transcriptional control of chondrocytes fate and differentiation. Birth defects research Part C: Embryo today: Reviews 2005;75(3):200-12.

23. Hallet SA, Ono W, Ono N. Growth plate chondrocytes: Skeletal development, growth and beyond. Int J Mol Sci 2019;20(23):6009-27.

24. Ahmed YA, Tatarczuch L, Pagel CN, Davies HMS, Mirams M, Mackie ES. Physiological death of hypertrophic chondrocytes. Osteoarthritis Cartilage 2007;15(5):575-6.

25. Bianco P, Cancedda FD, Riminucci M, Cancedda R. Bone formation via cartilage models.: The border line chondrocyte. Matrix Biology 1998;17(2):185-92.

26. Houben A, Kosdtanova-Poliakova D, Weissenböck M, Grat J, Teufel S, Mark K, Hartman Ch. Beta-catenin activity in late hypertrophic chondrocytes locally orchestrates osteoblastogenesis and osteoclastogenesis. Development 2016;143(20):3826-38.

27. Guilak F, Nims R, Dicks AM, Wu CL, Meulenbelt I. Osteoarthritis as a disease of the cartilage pericellular matrix. Matrix Biol 2018;7172:40-50.

28. Kao ZCh, Lee J, Huang HZ. Review article. What is new about the molecular genetics in matrix producing soft tissue tumours? The contributions to pathogenetic understanding and diagnostic classification. Virchows Arch 2020;476(1):121-34. 